\title{
Erratum to: Rats demonstrate helping behavior toward a soaked conspecific
}

\author{
Nobuya Sato ${ }^{1}\left[\right.$ Ling $\operatorname{Tan}^{1} \cdot$ Kazushi Tate $^{1} \cdot$ Maya Okada $^{1}$
}

Published online: 25 July 2015

(c) Springer-Verlag Berlin Heidelberg 2015

\section{Erratum to: Anim Cogn}

DOI 10.1007/s10071-015-0872-2

The authors would like to correct the word "less" to "more" in the sentence under the heading "Results and discussion". The correct sentence should read as:

This indicates that the soaked rats spent more time in the pool area than did the helper rats.

The online version of the original article can be found under doi:10.1007/s10071-015-0872-2.

Nobuya Sato

nsato@kwansei.ac.jp

1 Department of Psychological Sciences, Kwansei Gakuin

University, 1-1-155, Uegahara, Nishinomiya,

Hyogo 662-8501, Japan 